\title{
STEENROD OPERATIONS AND TRANSFER
}

\section{LEONARD EVENS ${ }^{1}$}

In this note I show that for the cohomology of a group with coefficients in $Z / p Z$, the transfer homomorphism commutes with the Steenrod reduced power operations.

Suppose that $G$ is a group and $H$ a subgroup of finite index. Let $P$ denote the cyclic group of prime order $p$ considered as a subgroup of $S_{p}$, the symmetric group on $p$ symbols. As in [2], we denote by $S S G$, the semi direct product of the permutation group $S$ with the group $G^{p}$ where the former acts on the latter by permuting the factors. Then $P \times G$, for example, may be considered a subgroup of $P S G$ by imbedding $G$ in $G^{p}$ via the $p$-fold diagonal map. If this is done, the basic Steenrod construction may be described simply: Given $\alpha \in H^{q}(G, Z / p Z)$, form the element $1 \int \alpha \in H^{p q}(P S G, Z / p Z)$ as follows. Let $X$ be a $G$-projective resolution of $Z$ and suppose $f \in \operatorname{Hom}_{G}\left(X_{q}, Z / p Z\right)$ represents $\alpha$. Let $W$ be a $P$-projective resolution of $Z$ with augmentation $\epsilon$. Then $W \otimes X^{p}$ becomes a $P S G$ projective resolution of $Z$ and $\epsilon \otimes f^{p}$ is a $P S G$ homomorphism of $W \otimes X^{p}$ into $Z \otimes(Z / p)^{p} \cong Z / p Z$ which is in fact a cocycle whose cohomology classdenoted by $15 \alpha$-depends only on $\alpha$. (More generally, we may replace $P$ by any subgroup $S$ of $S_{p}$, but then it is necessary to include a sign in the action of $S$ on $Z \otimes(Z / p Z)^{p}$.) Denote by $P(\alpha)=P_{G}(\alpha)$ $\in H^{p q}(P \times G, Z / p Z)$ the restriction of $15 \alpha$ to the subgroup $P \times G$. $P(\alpha)$ is the basic object from which the reduced powers are constructed.

Suppose next that $\beta \in H^{q}(H, Z / p Z)$. Let $T=\{\tau\}$ be a left transversal of $H$ in $G$. Since $H$ is a subgroup of $G, X$ is also an $H$-projective resolution of $Z$ and if $g \in \operatorname{Hom}_{H}\left(X_{q}, Z / p Z\right)$ represents $\beta$, then $\sum_{\tau \in T} \tau g$ represents $\operatorname{tr}_{H \rightarrow G}(\beta)$. (See [1, Chapter XII, §8].) We wish to study $P(\operatorname{tr} \beta)=\operatorname{res}\left(1 \int(\operatorname{tr} \beta)\right)$. As above, $1 S \operatorname{tr} \beta$ is represented by

$\epsilon \otimes\left(\sum \tau g\right)^{p}=\sum \epsilon \otimes \tau_{1} g \otimes \tau_{2} g \otimes \cdots \otimes \tau_{p} g \quad\left(\tau_{1}, \tau_{2}, \cdots, \tau_{p}\right) \in T^{p}$.

We decompose this sum into a sum of terms each of which is a $P \times G$ cocycle. To accomplish this end, let $P \times G$ act on $T^{p}$, the first factor by permutation, the second diagonally. Under this action $T^{p}$ decom-

Received by the editors July 28, 1967.

${ }^{1}$ This research was supported by NSF grant GP 5867. I should like to thank Gene Lewis who suggested the problem to me and who contributed to this paper several helpful remarks. 
poses into a set of disjoint orbits $\{0\}$. Also if a given orbit 0 contains $\left(\tau_{1}, \tau_{2}, \cdots, \tau_{p}\right)$ and $L$ is the subgroup of $P \times G$ fixing $\left(\tau_{1}, \tau_{2}, \cdots, \tau_{p}\right)$, then the contribution to the total sum from this orbit 0 represents the transfer from $L$ to $P \times G$ of some element of $H^{*}(L, Z / p Z)$.

Consider $P \cap L$. Suppose first that $P \cap L=(1)$. Then the product $P L=M$ is direct. We claim that the transfer from $L$ to $P \times L$ is trivial. If this contention is granted, it follows by transitivity that the transfer from $L$ to $P \times G$ is trivial, and the contribution from the given orbit is trivial. On the other hand, the claim itself is valid since everything in $H^{*}(L, Z / p)$ is the restriction of something in $H^{*}(P \times L, Z / p)$ and restriction followed by transfer is multiplication by the index which in this case is $p$.

Suppose instead, that $P \cap L=P$. However, the only elements of $T^{p}$ which are fixed by $P$ are those of the form $(\tau, \tau, \tau, \cdots, \tau)$ with $\tau \in T$, and the set of all these forms one orbit for $P \times G$. Moreover, in this case, taking $(\tau, \tau, \cdots, \tau)=(1,1, \cdots, 1)$, we have $L=P \times H$. Hence, the only (possibly) nontrivial contribution to the sum is the transfer from $P \times H$ to $P \times G$ of the element of $H^{*}(P \times H, Z / p Z)$ represented by $\epsilon \otimes g^{p}$, that is, the transfer of $P_{H}(\beta)=\operatorname{res}(1 S \beta)$.

Summarizing the above result in a formula, we have

Proposition 1. $\operatorname{tr}_{P \times H \rightarrow P \times G}\left(P_{H}(\beta)\right)=P_{G}\left(\operatorname{tr}_{H \rightarrow G}(\beta)\right)$.

To derive the desired result for reduced powers, we note that we can write $P_{G}(\alpha)=\sum \mu_{i} \times D_{G}^{i}(\alpha)$ where $\mu_{i}$ is an appropriate generator of $H^{i}(P, Z / p)$ and $D_{G}^{i}(\alpha)$ denotes the $i$ th reduced power. (See [3, Chapter $7, \S 3]$.) Also $P_{H}(\beta)=\sum \mu_{i} \times D_{H}^{i}(\beta)$. On the other hand, $\operatorname{tr}_{P \times H \rightarrow P \times G}(\mu \times \rho)=\mu \times \operatorname{tr}_{H \rightarrow G}(\rho)$ so that we get the desired result.

Theorem 2. Let $G$ be a group, $H$ a subgroup of finite index. For each prime $p$, the Steenrod reduced power operations commute with transfer from $H$ to $G$.

REMARk. To gather all the Steenrod operations within the fold, we note that it is a triviality that the Bockstein homomorphism commutes with transfer.

\section{REFERENCES}

1. H. Cartan and S. Eilenberg, Homological algebra, Princeton Univ. Press, Princeton, N. J., 1956.

2. L. Evens, $A$ generalization of the transfer map in the cohomology of groups, Trans. Amer. Math. Soc. 108 (1963), 54-65.

3. N. Steenrod, Cohomology operations, revised by D. B. A. Epstein, Annals of Mathematics Studies, No. 50, Princeton Univ. Press, Princeton, N. J., 1962. 